\title{
Traffic Safety through Driver Assistance and Intelligence
}

\author{
Heiner Bubb* \\ Institute of Ergonomics, Technische Universität München, Munich, D-85747, Germany
}

Received: $08-01-2011$
Accepted: $04-04-2011$

\begin{abstract}
Future mobility has to keep the safety level that is already achieved and shall even improve it. This paper reviews the research about driver behavior and assesses how far the work has proceeded in the field of assistance and intelligence, which includes: the vehicle's longitudinal and transversal dynamics, the driver's behavior in intersections, the driver's safety-based approaching behavior, the driving dependability model for identification of traffic accident causations, and the analysis method for investigating whether every error cause a traffic accident. As a highlight, this paper introduces in enough detail the technical development of driver assistance systems in BMW, Daimler, Audi, Citroen, Lexus, VW, Opel, Peugeot, Renault, Chevrolet, Saab and Bosch. Both the contributions of research work concerning driving behavior analysis and driver assistance systems have to be aligned with a permanently updated interaction within the system of driver, vehicle and road traffic environment.
\end{abstract}

Keywords: driving behavior; driver assistance systems; traffic safety; driving task

\section{Introduction}

Since there is the motorized traffic being, one tries to improve the road safety. In order to derive suitable measures worldwide records of accident statistics are kept. If still substantially technical inadequacy was at the beginning of the development triggers for traffic accidents with injuries and even fatal exit, today the result of all investigations is worldwide that traffic accidents are to be led back to more than $95 \%$ exclusively on the driver's behavior ${ }^{1}$. Therefore, already very early one has started to introduce traffic controls by legislative measures (priority rules, speed limits, regulations to the right of the different traffic participants, like trucks, passenger cars, motorcycles, cyclist and pedestrian), whose observance is supervised by the police. In addition on the basis of the statistical knowledge many training measures have been developed by which the drivers should be better enabled and motivated for save driving. But also on the technical side there were many developments introduced by which an easier and better controllability of the vehicles was achieved. The today's possibilities and the continuous progress of microelectronics and sensor technology and linked to that the feasibility of technical intelligence have enormously extended this technical beginning of road safety improvement. Today the driver's assistance systems by which the comfort, but also the driving safety should be improved are offered by many car manufacturers. However, the positive effect of these systems can become only effective if the drivers also accept the assistance systems. Therefore, it is necessary to come to terms with the driving task and the driving errors that are possible by it in order to estimate correctly the probable effect of assistance systems and to give impulses for new and carrying on developments by such research.

In order to understand, why accidents occur and by what means they can be avoided it is necessary to analyze more in detail the driving task. In most publication

${ }^{*}$ Corresponding author: bubb@1fe.mw.tum.de 
driving task is categorized in primary and secondary driving task. In this paper it is followed to a proposal of Geiser $^{2}$, who divided the driving task into three levels.

- The primary driving task is to keep the car on the course. This task is to be divided again in the hierarchically ordered subtasks navigation (finding the correct way between the actual position and the destination), guidance (in the actual situation the course must be laid in such a way that a touch with standing or moving objects is avoided) and stabilization (all the mental information that was created in the task before is now realized by operating the steering wheel and the control elements).

- By the secondary driving task all activities are subsumed that are necessary depending on the driving demands. Hereby can be divided between actions, be which the driver shows his intention like flashing, hooting and similar, and reactions, by which the driver has to react on changed external conditions (e.g . dimming and switching to full beam, use the wiper and also change the gear in a hand operated car). It is obvious that these kinds of tasks can be automated and by this the driver will essentially relieved.

- Tertiary tasks assign actions that have nothing to do with the driving task (e.g. activities, by which comfort shall be improved and all forms of communication).

By the secondary and in more extent by the tertiary tasks a loss of safety is given by distraction. An important contribution to safety can be achieved by automation of tasks with reaction character and by simplification of tasks with action character.

In order to improve the accident situation essentially, first of all the focus is to be directed to the primary driving task. Within the primary task the guidance task has the biggest potential for improvement by the application of assistance systems. In order to find the human problems, a normative behavior is to be defined. Defining this normative behavior it is to be divided between the transversal (keeping the car on the road and keeping a safe distances to traffic participants left and right of the driver) and longitudinal (keeping a safe speed and a safe distance to leading cars) operation of the car. In this connection the following observation is important: An essential part of the driver's task on the guidance level is the estimation of sensations on the one hand (e.g. distances and maximum possible accelerations) and the estimation of dynamic values of the car on the other hand (speed and acceleration). That means: safe behavior depends essentially on human ability of estimation. The question is: how good can the driver perform this task and how he can be supported by technical means?

This paper gives a short overview about topical research of the driver's behavior and its modeling as well as about the historical development of technical supports which have influence the road safety. Further the question is answered, why not every error causes an accident.

\section{Researches of Driver Behavior Analysis and Modeling}

To understand, why traffic accidents occur and by which means they can be avoided, it is necessary to analyze and to model the driver's behavior more in detail. Recently some important works were carried out by Wang, Popiv and Plavsic in which the driver's behavior was analyzed and was modeled. These works refer on controlling as of the transversal as of the longitudinal dynamic. Another consideration is directed on the complex situation in intersections ${ }^{6,9,13-16}$. Especially the model aiming on the safety, suggested by Wang intends to simplify the driving task by a suitable advanced driver assistance system (i.e. an automatic speed control system) in order to avoid vehicle accidents and in addition to help at the same time to improve the traffic capacity ${ }^{16}$. All researches make an important contribution to the understanding of the driver's behavior and the required technical support. Surely in future some of these results for the traffic accident avoidance and the control of the vehicle security will be applied.

\subsection{Research results concerning in longitudinal and transversal dynamics}

For the regulation of the transversal dynamics the actual orientation of the car in relation to the course of the road is essential. Nobody is able to keep this orientation exactly parallel to the course of the road. A measure to describe this deviation is the "Time to Line Crossing" (TLC). It can be calculated from the angle $\Delta \varphi$ between the orientation of the car and the road depending on the actual position $d$ of the car on the road, the breath $B_{c a r}$ of 
the car, the breath Blane of the road and the speed $\mathrm{v}$ of the car (Fig. 1). The formula for TLC is:

$$
T C L=\frac{\frac{1}{2} \cdot\left(B_{\text {lane }}-B_{\text {car }}\right)-d \cdot \operatorname{sign}(\Delta \varphi)}{v \cdot \sin \Delta \varphi}
$$

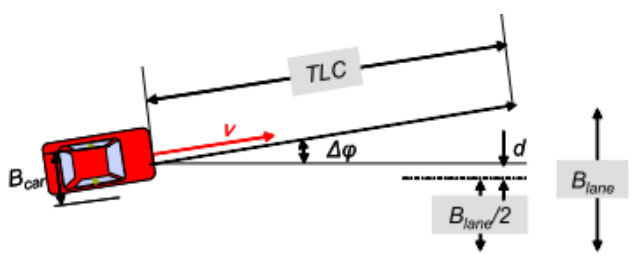

Fig. 1 Geometry defining the Time to Line Crossing (TLC)

From simulator research we found that in average the 15 th percentile TLC value is $3.7 \mathrm{~s}$. The standard deviation is in such an area that in the lower case the 15 th percentile TLC value is at $1.7 \mathrm{~s}$. That means that in few cases under the condition of a safety distance of 1 $\mathrm{m}$ and a transversal acceleration of $7 \mathrm{~m} / \mathrm{s}^{2}$ only a TLC of $0.9 \mathrm{~s}$ remains. That is too low for an adequate reaction.

For the case of the behavior in longitudinal direction Popiv et al. made real life experiments ${ }^{9}$. The experiments were carried out on the Autobahn with a car equipped with a radar based distance measurement system. In this case the Time to Collision (TTC) is the measure to describe the behavior. Its definition is shown in Fig. . The following car has a certain speed, here shown in a way-time-diagram. It follows with a certain distance, describable by the Time Gap (TGAP). The advice of safety experts is that this Time Gap should be in the best case $2 \mathrm{~s}$ and should not be lower than $1.2 \mathrm{~s}$. Depending on the lower speed of the preceding car it comes to a collision, described by the TTC value. Fig. 2 shows the observed TTC and TGAP values during the experiments described above. According to Fastenmeier $^{3}$ and van der Horst ${ }^{4}$ the following critical values can be defined: The minimal TGAP-value is 0.6 $\mathrm{s}$ (In the case of a value of 0.8 you will lose your driver license in Germany!). The critical TTC-value is at $5 \mathrm{~s}$. As can be seen from Fig. 3, in few cases unsafe and critical values can occur.

\subsection{Research results concerning the driver's safety-based approaching behavior}

In order to understand driver's safety-related approaching behavior during the car-following process, it is necessary to carry out a comprehensive analysis of vehicle-to-vehicle dynamic interactions. The emphasis of the work done by Wang is placed on the development of a driver's safety approaching behavioral model by considering the variability of following vehicle's speed to the leading vehicle's one and the relative distances between vehicles ${ }^{16}$. This model has been validated by using detailed vehicle trajectory data that was collected in naturalistic driving situations. The results show that the safety-based approaching behavioral model could be used to analyze driver's car-following behavior for a driving support and to reveal the essence of traffic flow characteristics at the microscopic level.



Fig. 2 Definition of the Time to Collision (TTC)

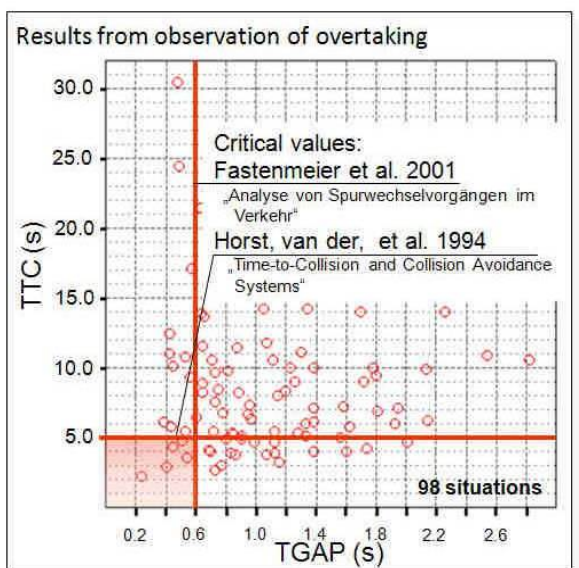

Fig. 3 Critical values for Time Gap an Time to Collision

In the simulation of driver's car-following behavior the original following distance was $25 \mathrm{~m}$, and the initial velocity was $20 \mathrm{~m} / \mathrm{s}$. The leading vehicle decelerated now within $4 \mathrm{~s}$ to $10 \mathrm{~m} / \mathrm{s}$. Fig. 4 shows how the speed and the distance of a pair of vehicles vary in a period of 60 s. As shown in Fig. 4, it is obvious that the distance finally oscillate in a narrow range between $13 \mathrm{~m}$ and 15 $\mathrm{m}$. This can be predicted by the developed model and in 
accordance with the famous typical 'close following spiral'. Besides, the time gap TGAP increases from the former value $1.25 \mathrm{~s}$ to the present $1.4 \mathrm{~s}$, which is well explained by the influence of the deceleration that causes the driver to pay more attention to the leading car and to be more careful by a larger time gap. The numerical results show the evidence that the developed model can describe the driver's safety approaching behavior rationally. The values are on the safe side according to the critical values of Fastenmeier ${ }^{3}$.
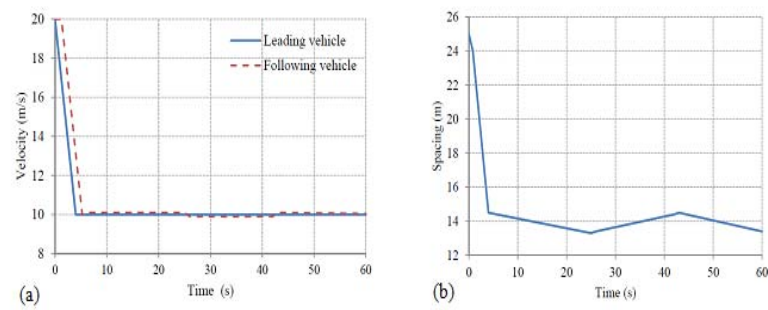

Fig. 4 Following speed and spacing with driving time while deceleration

\subsection{Research results concerning the driver's behavior in intersections}

In Europe, depending on country, intersections account for $30 \%$ to $60 \%$ of all injury-related accidents ${ }^{9}$. In order to understand driver's behavior in intersections, eyetracking research was carried out using the driving simulator of the Institute of Ergonomics (TUM) ${ }^{9}$. With the simulator it was possible to control the traffic situation as a function of the behavior of the experimental subjects. Thus it was guaranteed that every subject came into the same traffic situation.

In order to determine the intersection situations to be realized in the simulator, a comprehensive theoretical analysis of the most important influences on the driving task at an intersection was carried out. This resulted in a test course, which exists of 10 intersection situations. For it the maneuvers (on the left, right and left), the priority regulation (having priority of way, letting the right of way, stop sign, and right- pre-left regulation) and the presents of a car ahead was varied. The difficulty of task in every situation has been evaluated by the identification of the most important influences. In general, ideal driving behavior can be described as a behavior, which corresponds to legal regulations. Nevertheless, there is a span of the correct behavior for every traffic situation. A failure analysis is carried out by the comparison between the nominal and the actual driving behavior. In addition, the following values were analyzed by means of eye tracking: glance duration, glance frequency, percentage of time and maximum glance duration on 16 predefined Areas of Interest (AoI). The presence of other road users was the strongest Performance Shaping Factor (PSF) of the visual behavior. If more than four objects were present in the scene, the visual behavior was determined almost completely through the objects in the scene. This glance behavior was independent on the maneuver carried out or the priority regulation. The consequence was that in complicated scenarios the views in priority-entitled directions were often omitted. In Fig. 5 the difference between the glance sequences without and with other traffic is shown. In contrast to that the top down controlled glance behavior, which is characterized by an active searching, is dependent on given intersection characteristics. In this case the maneuver itself had stronger influence than the priority regulation. The fact is worth mentioning also that mostly the same subjects have committed the same error type, whether missing focusing on traffic signs or letting out of important partial task or the typical number of glances in certain phases. This supports the hypothesis that a high portion of errors is of systematic nature and could be so also prevented.

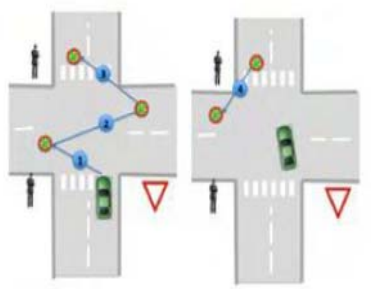

a) b)

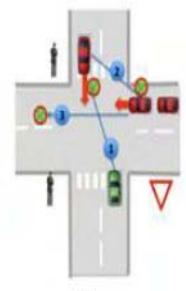

c)

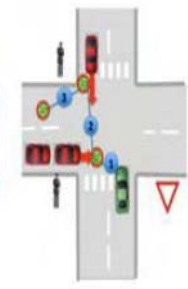

d)
Fig. 5 Typical look sequence in Scenario 6 for: a) Drive through if no other road users exist, b) Bending if no other road users exist, c) Drive through with oncoming and traffic crossing from the right, d) Drive through with oncoming and traffic crossing from the left

The task of the driver in intersections can generally be divided into the phases "approximating", "slowing down", "crossing", "bending" and "leaving the intersection". Typical errors can be observed in every of these phases 9 . The results show that the drivers can profit mostly from an assistance system which gives suitable support in the slowing down phase. An easy 
system with high potential would indicate a suitable speed to the driver already in the slowing down phase. Another support possibility is the reduction of the load by the support in the most exacting partial tasks. This is, for example, the knowledge about the momentary traffic regulation, above all in critical situations and in situations with a big number of other traffic vehicles. Therefore, an assistance system which indicates the driver the priority regulation in critical situations would relieve the driver clearly. This visualization could take place, e.g., in the Head-Up-Display. In this manner the priority of the most relevant information for the driver had already been realized. Therefore the sensors available in the vehicle and maps information could already deliver the necessary information in order to reduce the most dangerous errors during driving maneuvers in the intersection area. Such systems would promote the driver's competence. Nevertheless, accomplishing the action remained still at the driver. In comparison to available warning systems such an approach could raise the acceptance of the user and would be cost-effective, besides.

\subsection{Research results concerning the driving dependability model for identification of traffic accident causations}

Because daily driving behavior involves a seemingly endless variety of traffic situations, analysis and modeling of driving behavior have become increasingly important to traffic safety and capacity. However, many traffic accidents studies and databases applications offer only partial descriptions of driving errors. Therefore, it is almost certain that they are misapplied in identifying, measuring and assessing error-inducing driver behaviors. Owing to significantly individual differences in everyday driving behavior, it is quite difficult to assess the relative importance of driver errors compared with vehicle faults or road environment anomalies. Unlike previous studies about driving behavior modeling based on errors, Wang proposes a model-based methodology for driving dependability analysis ${ }^{13}$. The essence of modeling-based driving dependability analysis is concerned with understanding mechanisms of driving error in order to model them. Based on the model, driving dependability analysis will provide a way of collecting driving error data and of calculating driving reliability according to the data. As an example application, a driving dependability analysis of 2016 bus drivers at a public transport corporation driving under accident conditions has been conducted. The calculated results show that the driving dependability of $93.8 \%$ of drivers under accident condition is lowered to 0.99 . Therefore, the lower driving dependability for operating in a driver-vehicle roadway environmental system might result in traffic accidents. Based on the case study conducted, the model-based methodology could predict the effect of driving errors on road traffic safety in quantitative and qualitative terms.

\section{Analysis methods for investigating whether every error cause an accident}

As can be seen from Fig. 3, a certain amount of values could be observed that lay below the demanded values. The same results could be shown be the analysis of intersection behavior and the last paragraph. Why so few accidents occur in relation to it, when so often critical values can be observed in real life driving? Consequently, we need some analysis methods for investigating whether not every error cause an accident. The fault tree, event tree ${ }^{11}$ and the incident tree which proposed recently by $\mathrm{Wang}^{14}$ can be well used to analysis causations of traffic accidents. As a highlight, incident tree methodology including incident tree model, incident tree analysis method and mathematical simulation approach might be capable of identification of uncertain, random, complex, possible and variable characteristic of traffic accident occurrence.

\subsection{Event tree and fault tree analysis for traffic accident}

Reichart depicted the general pattern of an undesirable top event ${ }^{11}$, how is called the accident in the science concerning reliability. This pattern is called "event tree" (see Fig. 6). That an accident, a top event, can occur always a certain situation is necessary, for example a curve. The second precondition is a human error, for example to judge the curve wrong and to drive too fast or to turn the steering wheel too less. The third precondition is a conflict object, for example a soft shoulder of the road. That altogether makes the conflict. Normally the driver recognizes that he made a mistake. The top event can appear only when his avoidance action is insufficient, for example a too vehement reaction on the steering wheel, or when the avoidance reaction is missed totally. Both, the conflict and the failed avoidance lead to the top event. When we make 
now a very simple calculation for the probability of such a top event, we can assume a probability for curves of 0.5 and in general a probability of $10^{-2}$ for the conflict object. The human error for well exercised action is at $10^{-3}$. That means the probability for a conflict is in the area of $10^{-6}$. As avoidance actions are more rare activities, here the probability is one exponent higher. So the overall probability for a top event is in the area of $10^{-7}$. In order to understand this, let us convert this value in a value for that we have experience. According to the psychologist Pöppel ${ }^{10}$ one action unit needs in the case of highly skilled activities approximately $3 \mathrm{~s}$. That means that - assumed that every thousand action is wrong - the mean time between failures is at $50 \mathrm{~min}$. But not every error leads to the top event. When we assume that a driver every day uses his car for 1 hour, the mean time between events is at 23 years. That corresponds to our experience.

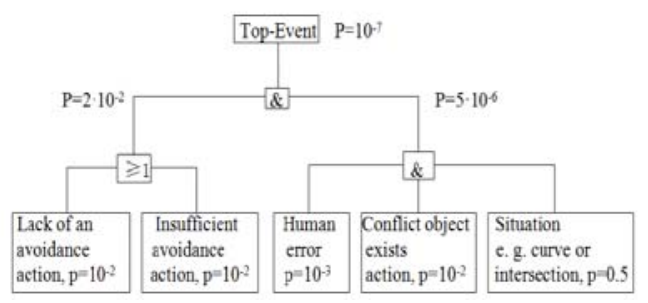

Fig. 6 General scheme for occurrence of accidents

Fig. 7 shows such an event or fault tree for the event "driving accident by street or weather conditions", also called "vehicle leave roadway accident". For this case we have some possible situations as, straight road, low friction coefficient, and human errors, like no adequate speed adaptation, a curve, no speed adaptation to the course or radius, reduced sight, no speed adaptation to the sight conditions, a deviation from the road or falling in sleep. Again we assume some probabilities for the occurrence of the situations and take known probabilities for human error. That leads to a probability for conflicts of $2 \cdot 10^{-2}$ per vehicle and year. As we not calculated here the probability of the conflict object and the insufficient success of avoidance actions, we can subsume this under the transitional probability to a traffic accident.

According to the research of Reichart ${ }^{11}$ this lies very constant for different top events at $10^{-4}$. The result is an accident likelihood of $3.2 \cdot 10^{-6}$ per vehicle and year. This agrees very well with the accidents statistics in Germany. As we have now a sufficient exact model for the occurrence of the top event, we can take from it the main influences in order to derive avoidance measures. We can calculate the importance. From this we can derive the necessity of assistance systems. The most effect is reached by lane departure assistance. That helps also for the tiredness accidents. A further advantage could be achieved by a friction coefficient indicator, which objectifies the road condition to the driver. Of course by ergonomic demands this information should be indicated in a form that can be understood in actual situation immediately by the driver. According to the research of Reichart ${ }^{11}$ this lies very constant for different top events at $10^{-4}$. The result is an accident likelihood of $3.2 \cdot 10^{-6}$ per vehicle and year. This agrees very well with the accidents statistics in Germany. As we have now a sufficient exact model for the occurrence of the top event, we can take from it the main influences in order to derive avoidance measures. We can calculate the importance. From this we can derive the necessity of assistance systems. The most effect is reached by lane departure assistance. That helps also for the tiredness accidents. A further advantage could be achieved by a friction coefficient indicator, which objectifies the road condition to the driver. Of course by ergonomic demands this information should be indicated in a form that can be understood in actual situation immediately by the driver.

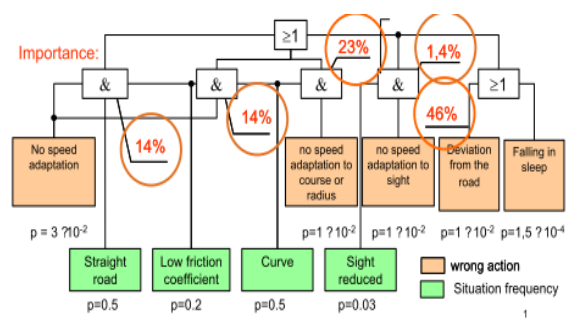

Fig. 7 Fault tree for the case "driving accident by street course

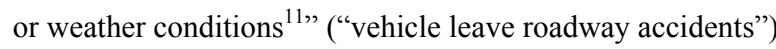

\subsection{Incident tree analysis for traffic accident}

Fault tree analysis (FTA) could apply to traffic safety analysis, but it often suffers from a lack of enough probabilistic basic events to check the consistency of the logic relationship among all events through linkage with gates. Sometimes, even logic relationship among all events is difficult to determine, and events in traffic system operation may have been experienced rarely or not at all. FTA methods can only identify the random characteristic of traffic accident(with probability), 
complex characteristic of accident(with human factor), possible characteristic of accident(with fuzzy set), variable characteristic of accident(with time requirement). However, a highlighted characteristic of traffic accident is uncertainty that cannot capture with FTA.

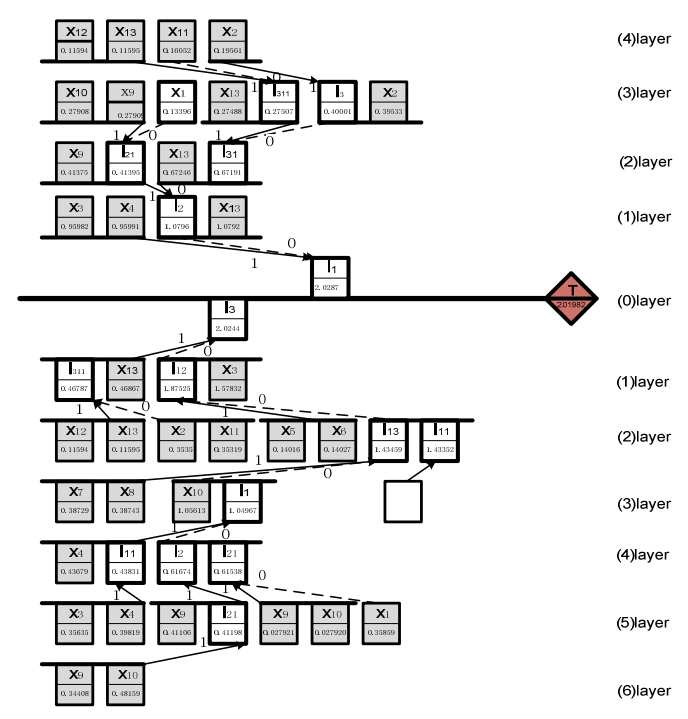

Fig. 8 Incident tree model of vehicle leave roadway accident In order to address the limitations, according to the definition of events, incidents, accident and functional analysis of relationship among them, from probability statistics to fuzzy information quantities of incidents and accident, Wang proposed an Incident Tree Analysis methodology (ITA) for filling in some gaps in FTA and Quantified Risk Assessment(QRA) by introducing fuzzy logic and information flow approaches ${ }^{14}$. Because the amount of information of an incident occurrence is related to uncertainty, the novel incident tree methodology that characterizes the information flow in a system instead of the logical relationship among events, and the amount of information of an incident instead of the probability of a basic event. The case study of "vehicle leave roadway accident" with ITA illustrates that the proposed methodology may not only capture the essential information transformations of accident that occurs in system operation, but also determine the various combinations of vehicle faults, and driver errors that could result in the occurrence of specified undesired incident at the system level even accident (Fig. 8).

\section{Technical development of Driver Assistance Systems}

As already shown in the introduction, every measure which simplifies car driving, is also a contribution to safe driving, because it avoids unnecessary distraction and attachment of mental concentration on contents not relevant for traffic. The technical development of the car is marked beside the progress of efficiency and power and beside the progress of the so-called passive safety, which has the protection of the passengers after an accident to the aim, in different extent by this form of the active safety, which should avoid a possible accident before it could take place. One can distinguish in this development the period of the purely mechanical measures from those of the electronic measures, which are able to realize a certain degree of technical intelligence. In the following these both ropes of development will be briefly outlined.

\subsection{Driver's support by mechanical assistance}

After the first consolidation phase in the period after the invention of the gasoline driven motor car in the 20 years of the last century diverse functions of the engine were automated (in this connection should be mentioned that for the earliest steam engines even the opening and closing of the valves that let flow the steam to the working cylinder was operated by human action! It was the invention of James Watt to control the valve operation by the rotation of piston driven crank axis and that brought the breakthrough of the steam engine). Best known is the introduction of the automatic - that means mechanical controlled - ignition timing in the twenties. Before, the driver had to adjust by ear the correct trigger time-point by a separate crank on the steering wheel. Of course this automation simplified the operating of the car and made it possible to draw more attention to the traffic. The demands of traffic, which become successively denser, was and is the driving force to develop further technical equipment that should simplify operating the car and make it more comfortable. In the 30 years the automatic transmission was invented and during the 50 years it was broadly introduced in cars of USA. In connection with the increasing car density in Europe, especially in Germany, in the 80 and 90 years the automatic transmission became also here very convenient. In the 60 and 70 years under the condition of the cars becoming heavier diverse steering function were technically supported. In this connection especially is to call the brake booster that use the negative pressure in the crank case for amplifying the 
foot pressure and the steering booster that use the hydraulic pressure prepared by the engine power. These two servo systems make car operating not only more comfortable but also faster and give so the opportunity to react faster. However, by this development the end of the mechanic-hydraulic supporting systems was reached. Already the next step brought electronics (see 4.2).

But not only the interaction between the driver and control elements that influence driving activities directly was influenced by the course of technical development but also the kind of information about the technical status and the moving parameters. By technical reasons thermometer - first directly mounted on the cooler was installed and some level indicator for the different oil circuits. Very soon the legislator demanded an indicator for speed. Also these instruments were ad-on instruments (a relic of this kind of thinking you can find in Citroen $2 \mathrm{CV}$ cars of earlier years!). Later in the end of the twenties and in the beginning of the thirties all these instruments were together with some bottoms and levers integrated in the dashboard.

\subsection{Driver's support by electronic assistance}

In the beginning of the 70 years the Antilock Braking System (ABS) was presented first time by MercedesBenz and shortly after by BMW. By this system during breaking the wheel speed is measured by electric sensors for every of the four wheels independently. When on one (or more) of these wheels no rotation is measured the corresponding valve is opened very fast and closed very fast again when the wheel is rotating again. Only by quick electronic circuits this procedure can be actuated so fast that the effective braking distance becomes not longer than in the case of locking brakes. In opposite to the locking wheels the turning wheel can transfer transversal forces and so the car even in the case of an emergency braking remains able for steering. Today nearly every new produced car is equipped worldwide with this system. A further consequent step was to integrate also the propulsion system in this control. A first application was the antiskid system, by which in the case of wheel spin the power of the engine is reduced. However, the Electronic Stability Program (ESP) of Bosch, first applied by Daimler-Chrysler, brought the breakthrough, well known by the "moose test". ESP uses the electric measured information of steering angel, accelerator position and brake pressure in order to calculate by computer's aid the ideal bend that is geometrically and physically defined by this information. This ideal bend is compared with the real bend calculated from the information of measured change of yaw angle and transversal acceleration. In the case the difference is over a predefined limit, motor power is reduced and the wheels are braked separately independent of each other (here exist various systems: in the more simple case the wheels of one car side are connected to each other; in a more elaborated system one side and one wheel of the opposite side are activated in the opposite direction). The preliminary last step in this development is the socalled active steering system of BMW. In the case of responding ESP independent of the reaction of the driver the steering system is influenced. By this system the effect of ESP is enlarged importantly ${ }^{7}$.

With the beginning of the $21^{\text {st }}$ century a further important step was done in introducing electronic in the interaction between driver and car. By the ACC (Automatic Cruise Control) not only like the conventional cruise control the predefined speed is kept by an electronic realized control system but also the distance to the car ahead. This distance is measured by a radar sensor. When the distance becomes too short not only the motor power is reduced but also the brakes are activated. By Automatic Cruise Control system the driver can keep the distance without own activity. That means a first step is done to automatic driving. In the first approach the system was not able to brake done until still stand. However, new developments of near region radar or infrared technique allow realizing even this option. In the described form ACC can be seen in a certain manner as a comfort system although it avoids unsafe distance to the car ahead. A very strong safety system is the emergency brake system (Bracing Assistance, BAS) first presented by Lexus and nowadays presented in an improved version by Mercedes-Benz. This system uses the same sensors as the described ACC. However, it calculates the approaching speed and in the case of high speed difference between the own and the leading car $3 \mathrm{~s}$ before a possible crash the systems gives a warning signal, $1.5 \mathrm{~s}$ before a possible crash it brakes autonomously with moderate deceleration and $0.6 \mathrm{~s}$ before a crash it initiates an emergency braking. It is obvious that these described systems give an important contribution to avoid longitudinal accidents. 
Independent of this development the technical observation of the transversal dynamic of the car was introduced. A very simple version is realized by Citroen in the $\mathrm{C} 4$ and $\mathrm{C} 5$. In this case by infrared beamer and sensor the leading lines on the road are observed. In the case of over climbing the line this is indicated to the driver by a vibrator on the corresponding side of the seat. The Heading Control (HC) of BMW and similar of Daimler and Audi - developed within the European PROMETHEUS-project in the 80 years - observes the road ahead by a camera system and calculates from information derived from the camera picture the ideal steering wheel angle. In the case the driver does not keep this angle that is indicated by vibration on the steering well. Beside the already described measurement systems additionally video techniques and ultra sound (Bosch) will be used in order to observe the field round the car - even 3-dimensional information can be obtained by such techniques (Audi) and to derive from such information accident avoiding actuations. All these measures avoid the transversal accidents and give a significant contribution to demands formulated by Wang $^{15}$. Fig. 9 gives an overview on the described and to be expected development of driver assistance systems in near future.

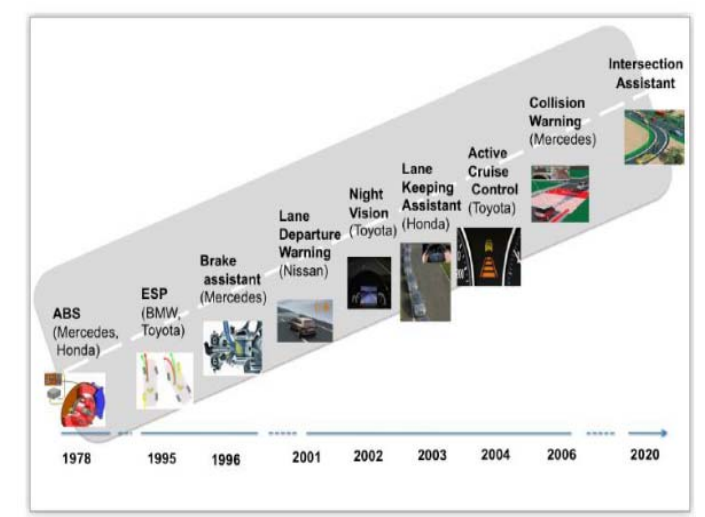

Fig. 9 The development of driver assistance systems

However, electric and later electronic gave also an important push to the instrumentation of cars. E.g. in the thirties of the last century a radio was not installed. It was foreseen for entertainment and seemed to have nothing to do with the serious task of driving (still in the fifties it was discussed seriously, if listening radio during driving is too much distracting!). However, the increasing demands for comfort made the incorporation of radios in car necessary. Today it contributes an essential part to traffic safety by traffic news. As the radio became such a central equipment for increasing comfort devices step by step it took over also other tasks. The most important was the integration of navigations systems. The radio was now equipped with a screen. The further development was to use this screen for additional tasks. A big and important step was introduced 2003 by the 7-series of BMW with the socalled "I-Drive". Now nearly all functions could be operated by an innovative central control element. By this control element 8 ground menus can be selected by moving it on the diagonals of eight angular. Within the selected menus a choice can be made by turning the control element. Pushing the controller activates the selected function. The BMW I-Drive was an excellent example for other car manufacturers. Very soon Audi showed a similar system, called MMI, Mercedes followed with its S-class. Meanwhile also criticism in this concept emerged. Many manufacturers put instead of on the central control element on the touch screen technology (e.g., Lexus, VW) or remain on the conventional button operation (Opel, Peugeot / Citroen, Renault). In general this technological development led to an organization of driver's working place that nowadays is very convenient within the automotive industry. It is separated between the DIS (driver information system) and the DAS (driver assistant systems). The DIS is located in the centre of the dashboard on the former position of the radio. It contains the functions radio, CD-player, sometimes TV, the operation of the integrated phone, newly even e-mail and internet but also the operation of the climate system and the navigation system. Furthermore also some general adjustments of the car, like different functions of ESP, chance of the suspension system and similar can be operated within this DIS.

The DAS is located central to the driver. It contains all functions supporting driving (e.g. ACC). Most indications are done in the instrument behind the steering wheel und newly also in the HUD (Head-UpDisplay, see Chevrolet Corvette, BMW 5- and 6- series, Citroen C6, Peugeot 5008, Saab 9-5). The control element for driving and operating the DAS are arranged round the steering wheel. Meanwhile it is also convenient to locate the light functions in the area left of the steering wheel. So a certain standard of car operation has been established. Also this is a contribution to active safety. 


\section{Summary and conclusions}

As shown Driver Assistant Systems that could help against transversal and especially longitudinal accidents exist already in form of the so-called Heading Control system, in form of the Active Cruise Control (ACC), and the emergency brake system (BAS). Today different affords are made to work for pedestrian protection and to give aids in the intersection situation. However, one problem is not solved until now. It seems to be necessary to connect these systems with the fun of driving. A research of Sacher has shown that even possessors of such systems (in this case ACC) don't use it in the daily driving ${ }^{12}$. The main reason is that they don't understand correctly the function. A further reason is that they feel to be treated like a child and that the observation of these systems make more effort than not to use it. It is a big demand for ergonomics to present solution proposal for this dilemma.

In this contribution the latest achievements in the research of driver behavior analysis and modeling have been generally reviewed and could help for a heuristic understanding the state of art in traffic safety. We can see the effort made not only by the scholars in ergonomics, psychology, engineering, but also by the scholars from interdisciplinary combinations. The researches give different sights to investigate and assess the drive behavior in accordance with the vehicle kinematics, the intrinsic features of the drivers, the mathematical solutions, etc. It is instructive to note the results proposed. There is the hope that by an integrated approach the performance of driver assistance systems can support increasing. Traffic accidents and vehicle safety make driver assistance systems necessary, as the brief view of the technical development of driver assistance systems has shown. Although we can hardly assert how far the driver assistance systems can contribute to the road traffic safety, the researchers and engineers will continuously endeavor to create a road user-friendly environment through various driver assistance systems. No doubt so much, advanced driver assistance systems do help against transversal and especially longitudinal accidents.

\section{References}

1. Bubb H, Driver behavior and assistance, Journal of Beijing Institute of Technology,19(S2) (2010),1-22

2. Geiser G., Mensch-Maschine-Kommunikation im Kraftfahrzeug, ATZ 87, 1985 77-84
3. Fastenmeier, W., Hinderer, J., Lehnig, U., Gstalter, H., Analyse von Spurwechselvorgängen im Verkehr. Zeitschrift für Arbeitswissenschaft, 2001/1 (55). S. 15-23

4. Horst, van der, R., Hogema, J.,Time-to-Collision and Collision Avoidance Systems. Salzburg: Proceedings of the 6th ICTCT Workshop: Safety Evaluation of Traffic Systems 1990. S. 109-121

5. Intersafe, User needs and operational requirements for cooperative intersection safety system (European Union project, 2009)

6. Lange, C. Arcati, A., Bubb, H., and Bengler, K., Haptic gear shifting indication: Naturalistic driving study for parametrization, selection of variants and to detremine the potential for fuel consumption reduction, in AHFE International Conference, Session 65, Application of Cognitive Ergonomics. Miami, 2010

7. Langen, P., Vom Fahrwerk zum Fahrerlebnis, Paradigmenwechsel, in Vortrag auf der fahrwerk.tech, Tagung(TÜV Automotive GmbH, TÜV Akademie GmbH TÜV SÜD Gruppe, München ,2005)

8. Plavsic, M., Klinker, G., Bubb, H., Situation Awareness Assessment in Critical Driving Situations at Intersections by Task and Human Error Analyses, Human Factors and Ergonomics in Manufacturing \& Service Industries, 20(3)(2010), 177-191

9. Popiv, D., Rakic, M., Nestler, S., Bengler, K., Bubb, H. , Timing concept for assistance of anticipatory driving, in Proc. 17th World Congress on Ergonomics (IEA, Beijing, 2009)

10. Pöppel, Grenzen des Bewusstseins - Wie kommen wir zur Zeit und wie entsteht Wirklichkeit? (Insel Taschenbuch, Frankfurt a.M. und Leibzig,2000)

11. Reichart, G., Menschliche Zuverlässigkeit beim Führen von Kraftfahrzeugen. Fortschritt-Berichte, Reihe 22, Mensch-Maschine-Systeme, (Nr. 7, VDI-Verlag, Düsseldorf, 2001)

12. Sacher, H., Gesamtheitliche Analyse des Bedienverhaltens von Fahrzeugfunktionen in der täglichen Nutzung, (Dissertation an der Technischen Universität München,2008)

13. Wang, W., Cao, Q., Ikeuchi, K., Bubb, H., Reliability and safety analysis methodology for identification of drivers' erroneous actions, International Journal of Automotive Technology, 11(6) (2010),873-881

14. Wang, W. Jiang, X., Xia, S. Cao, Q., Incident tree model and incident tree analysis method for quantified risk assessment: an in-depth accident study in traffic operation, Safety Science, 48(10) (2010),1248-1262

15. Wang, W., Hou, F., Tan, H. Bubb, H., A framework for function allocation in intelligent driver interface design for comfort and safety, International Journal of Computational Intelligence Systems, 3(5) (2010),531-541

16. Wang. W., Zhang, W., Guo, H., Bubb, H., Ikeuchi, K., A safety-based behavioral approaching model with various driving characteristics, Transportation Research Part C, doi:10.1016/j.trc.2011.02.002 